\section{Glucagon-like peptide-1 and satiety}

On the basis of their observation that intracerebroventricular administration of glucagon-like peptide-1 (residues 7-36) amide (GLP-1) reduced food intake in rats, Turton et al. ${ }^{1}$ suggest that GLP-1 is a physiological mediator of satiety. Using c-Fos immunohistochemistry as a marker of neuronal activity, they reported that GLP-1 induces c-Fos expression "exclusively" in the paraventricular hypothalamus and central amygdala, regions of the brain important in the regulation of feeding. Although consistent with the hypothesis that GLP-1 causes satiety, there are alternative interpretations for the earlier result. We report here that GLP-1, in addition to reducing food intake ${ }^{1,2}$, also supports the development of a strong conditioned taste aversion, indicating that GLP-1 has aversive side-effects that could attenuate consumption and hence provide one explanation for the data of Turton and colleagues'.

We administered GLP-1 $(0.3,1.0$ or $3.0 \mu \mathrm{g})$ intracerebroventricularly to undeprived rats at the beginning of the dark phase of their light-dark cycle. Consistent with ref. $1,3.0 \mu \mathrm{g}$ was the lowest dose to produce reliable reductions in food consumption over a 2 -hour period (Fig. 1a). To examine the potential of GLP-1 to produce a conditioned taste aversion, we infused saccharin directly into the oral cavity of other rats ${ }^{3}$, followed immediately by GLP-1 administration $(1.0 \mu \mathrm{g}$ or $3.0 \mu \mathrm{g})$. When re-exposed to saccharin several days later, a significant number of rats rejected the taste when it was paired with $3.0 \mu \mathrm{g}$ but not 1.0 $\mu \mathrm{g}$ of GLP-1 (Fig. 1b). Rats that received saccharin that had previously been paired with intraperitoneal injection $\left(63 \mathrm{mg} \mathrm{kg}^{-1}\right)$ of the emetic agent lithium chloride displayed a conditioned taste aversion similar in magnitude to $3.0 \mu \mathrm{g}$ GLP-1 (data not shown). Saccharine exposure followed by administration of control solutions for GLP-1 or LiCl did not cause rats to reject saccharin during testing for conditioned taste aversion, nor did exposing rats to GLP-1 or LiCl that was not previously paired with saccharin. Thus, reduction of food intake by GLP-1 is apparently accompanied by symptoms sufficient to support a conditioned taste aversion.

We have also examined the pattern of c-Fos induction after GLP-1 administration. Consistent with ref. 1, we find that c-Fos is expressed in the paraventricular nucleus and central amygdala ${ }^{2}$. GLP-1 also induces strong c-Fos expression in the nucleus of the solitary tract, area postrema and pontine parabrachial nucleus, eliciting a pattern of brainstem activity similar to that elicited by $\mathrm{LiCl}^{3}$.

In summary, we find that intracerebroventricular GLP-1 generates neural activity in the brainstem, as assessed by c-Fos immunohistochemistry ${ }^{2}$, strikingly similar to that of a known emetic agent ${ }^{3}$. We have also found that the lowest dose of GLP-1 to reduce food intake supports
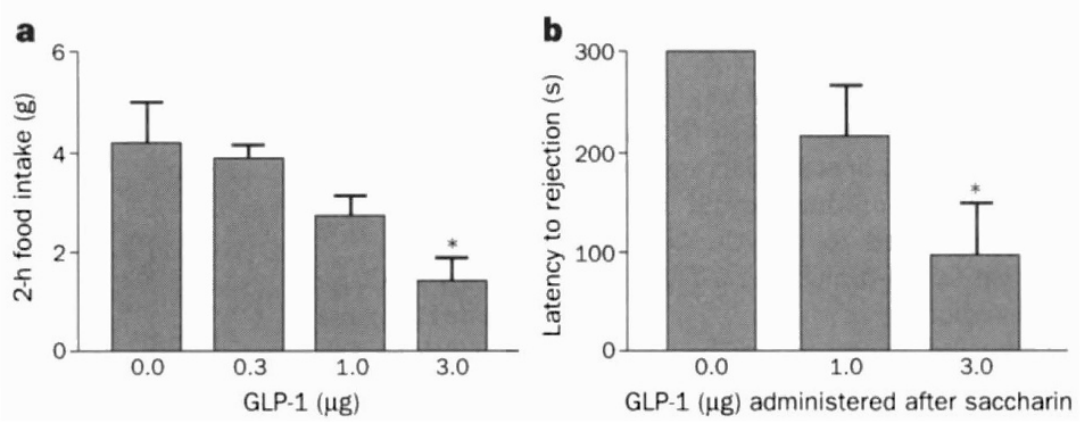

Figure 1 a, Food intake in undeprived rats $2 \mathrm{~h}$ after infusion of synthetic cerebrospinal fluid (sCSF; $n=6$; left bar) or GLP-1 $(0.3 \mu \mathrm{g}, n=7 ; 1.0 \mu \mathrm{g}, n=6 ; 3.0 \mu \mathrm{g}, n=4)$ just before the dark phase $\left(F_{3,19}=4.27\right.$, $P<0.05$; GLP-1 versus SCSF; $\left.{ }^{*}, P<0.01\right)$. b, Latency to reject intraoral infused saccharine solution $(2.5 \mathrm{ml}$ of $0.15 \%$ over $5 \mathrm{~min})$ by undeprived rats that had previously received saccharin immediately before receiving $\operatorname{SCSF}(n=5)$ or GLP-1 $(1.0 \mu \mathrm{g}, n=6 ; 3.0 \mu \mathrm{g}, n=5)$. Rats more rapidly rejected saccharin paired with $3.0 \mu \mathrm{g} \mathrm{GLP}-1\left(F_{2,13}=5.17, P<0.05 ; 3.0 \mu \mathrm{g} \mathrm{GLP}-1\right.$ versus $\left.\mathrm{SCSF} ;{ }^{*}, P<0.05\right)$. Male Long-Evans rats $(300-350 \mathrm{~g})$ were housed individually in stainless-steel cages and given free access to food and water. Cannulae were positioned and assessed as described elsewhere ${ }^{2,3}$. Intracerebroventricular drugs were dosed in a $3.5-\mu \mathrm{l}$ volume. Immediately after saccharine exposure, rats were given either GLP-1 $\left(1.0 \mu\right.$ g or $3.0 \mu$ g; paired GLP-1 group), $\mathrm{LiCl}\left(63 \mathrm{mg} \mathrm{kg}^{-1}, 0.15 \mathrm{M}\right.$; paired LiCl group) or the proper controls of both treatments (sCSF; unpaired GLP-1 or isotonic saline; unpaired $\mathrm{LiCl}$ ). Several days later and without exposure to saccharin, rats in paired groups received control treatments whereas unpaired rats received GLP-1 $(3.0 \mu \mathrm{g})$ or $\mathrm{LiCl}$. Thus all rats had equal exposure to drugs and to saccharin, either on the same day (paired groups) or on separate days (unpaired). Finally, latency to reject intraoral saccharin was assessed (active fluid expulsion and/or passive dripping from the oral cavity). the development of a conditioned taste aversion. These observations suggest that nonspecific, aversive symptoms may be elicited by GLP-1 and that these symptoms could account for observed reductions in food intake. It has often been cautioned that hormonal and pharmacological treatments can lead to reductions in food intake for various reasons, and that criteria for classifying satiating agents need to exclude other possible, nonspecific factors (see, for example, ref. 4). Our observations argue for a more careful consideration of GLP-1 before classifying it as a satiating agent.

G. van Dijk, T. E. Thiele

R. J. Seeley, S. C. Woods

I. L. Bernstein

Departments of Psychology and Medicine,

University of Washington,

Seattle,

Washington 98195, USA

e-mail:gvandijk@u.washington.edu

Bloom et al. reply - van Dijk et al. provide interesting and valuable new data. But exogenously administered neurotransmitters penetrate a wide area in an abnormal concentration gradient. They probably activate multiple circuits simultaneously, particularly when at grossly supraphysiological concentrations.

In contrast, we based our observations on the administration of a specific antagonist that acts only to block endogenous peptide release and thus affects only physiologically active circuits. Blocking endogenous GLP-1 release causes healthy animals to eat more. Since the publication of our paper ${ }^{1}$, we have administered the GLP-1 antagonist exendin (residues 9-39) to rats twice daily for 10 days; the animals not only significantly increase their $24-\mathrm{h}$ food intake but also gain a statistically significant amount more weight than controls. Our view therefore remains that endogenous GLP-1 is indeed involved in the regulation of satiety, irrespective of its other possible functions.

\section{S. R. Bloom $\dagger$}

Division of Endocrinology and Metabolism, Hammersmith Hospital Medical School,

London W12 ONN, UK

e-mail:sbloom@rpms.ac.uk

1. Turton, M. D. et al. Nature 379, 69-72 (1996).

2. van Dijk, G, et al. Am. J. Physiol. 271, R1096-R1100 (1996).

3. Swank, M. W., Schafe, G. E. \& Bernstein, I. L. Brain Res. 673, 251-261 (1995).

4. Deutsch, J. A. in Handbook of Behavioral Neurobiology Vol, 10: Neurobiology of Food and Fluid Intake (ed. Stricker, E. M.)

151-182 (Plenum, New York, 1990).

† On behalf of the coauthors of ref. 1 .

scientific correspondence is intended to provide a forum in which readers may raise points of a scientific character. Priority will be given to letters of fewer than 500 words and 10 references. 